\title{
A method for histopathological study of the multifocal nature of spinal cord lesions in murine experimental autoimmune encephalomyelitis
}

Katherine N. Gibson-Corley, Alexander W. Boyden, Mariah R. Leidinger, Allyn M. Lambertz, Georgina Ofori-Amanfo, Paul W. Naumann, J. Adam Goeken, Nitin J. Karandikar

Experimental autoimmune encephalomyelitis (EAE) is a well-established mouse model for multiple sclerosis and is characterized by infiltration of mononuclear cells and demyelination within the central nervous system along with the clinical symptoms of paralysis. EAE is a multifocal and random disease, which sometimes makes histopathologic analysis of lesions difficult as it may not be possible to predict where lesions will occur, especially when evaluating cross sections of spinal cord. Consequently, lesions may be easily missed due to limited sampling in traditional approaches. To evaluate the entire length of the spinal cord while maintaining anatomic integrity, we have developed a method to section the cord within the decalcified spinal column, which allows for the study of the multifocal nature of this disease and also minimizes handling artifact. HE and Luxol fast blue staining of these spinal cord sections revealed a paucity of lesions in some areas, while others showed marked inflammation and demyelination. The percentage of spinal cord affected by EAE was evaluated at four separate areas of longitudinally sectioned cord and it varied greatly within each animal. Immunohistochemical staining of in situ spinal cords which had undergone decalcification was successful for key immuno-markers used in EAE research including CD3 for T cells, B220 for B cells and F4/80 for murine macrophages. This method will allow investigators to look at the entire spinal cord on a single slide and evaluate the spinal cord with and without classic EAE lesions. 
1 A method for histopathological study of the multifocal nature of spinal cord

2 lesions in murine Experimental Autoimmune Encephalomyelitis

3

4

5 Katherine N. Gibson-Corley, Alexander W. Boyden, Mariah R. Leidinger, Allyn M.

6 Lambertz, Georgina Ofori-Amanfo, Paul W. Naumann, J. Adam Goeken, Nitin J.

7 Karandikar

8

9

10 11 lowa 52242 USA

12

13

14

15

16

17

23

24

25

26

27

Department of Pathology, University of lowa Carver College of Medicine, lowa City,

\section{CORESPONDING AUTHOR:}

Katherine N. Gibson-Corley

Assistant Professor, Pathology

1167ML, 200 Hawkins Drive

Roy J. and Lucille A. Carver College of Medicine

University of lowa

lowa City, lowa 52242

Phone: 319-384-4430

Email: katherine-gibson-corley@uiowa.edu 


\section{ABSTRACT}

Experimental autoimmune encephalomyelitis (EAE) is a well-established mouse

model for multiple sclerosis and is characterized by infiltration of mononuclear cells and demyelination within the central nervous system along with the clinical symptoms of paralysis. EAE is a multifocal and random disease, which sometimes makes histopathologic analysis of lesions difficult as it may not be possible to predict where lesions will occur, especially when evaluating cross sections of spinal cord.

Consequently, lesions may be easily missed due to limited sampling in traditional approaches. To evaluate the entire length of the spinal cord while maintaining anatomic integrity, we have developed a method to section the cord within the decalcified spinal column, which allows for the study of the multifocal nature of this disease and also minimizes handling artifact. HE and Luxol fast blue staining of these spinal cord sections revealed a paucity of lesions in some areas, while others showed marked inflammation and demyelination. The percentage of spinal cord affected by EAE was evaluated at four separate areas of longitudinally sectioned cord and it varied greatly within each animal. Immunohistochemical staining of in situ spinal cords which had undergone decalcification was successful for key immuno-markers used in EAE research including CD3 for T cells, B220 for B cells and F4/80 for murine macrophages. This method will allow investigators to look at the entire spinal cord on a single slide and 47 evaluate the spinal cord with and without classic EAE lesions. 


\section{INTRODUCTION}

53

54

55

56

57

58

59

60

62

64

65

66

67 71 future therapeutics in this model. Indeed, researchers' ability to efficiently and

72

Multiple sclerosis (MS) is a debilitating autoimmune disease characterized by cellular inflammation into - and the progressive demyelination of - the central nervous system (CNS), subsequently leading to a multitude of clinical symptoms (Sospedra \& Martin 2005; Steinbach \& Merkler 2014; Trapp \& Nave 2008). Experimental

autoimmune encephalomyelitis (EAE) is a well-studied mouse model of MS-like disease due not only to its convenience for immune system manipulation, but importantly because it recapitulates various hallmarks of human disease such as CNS inflammation and paralysis (Constantinescu et al. 2011; Duffy et al. 2014; McCarthy et al. 2012). EAE can be induced by an assortment of immunizations, but most often includes the subcutaneous injection of peptides derived from neuroantigens (such as myelin basic protein, myelin proteolipid protein, or myelin oligodendrocyte glycoprotein (MOG)) as part of an emulsion with Complete Freund's Adjuvant (CFA). In this study, we induced EAE disease in C57BL/6 mice with the commonly utilized $\mathrm{MOG}_{35-55}$ peptide. Upon disease onset of this classic model, the manifestation of clinical symptoms ensues, which is characterized by a grading scale of ascending paralysis (Ortega et al. 2013; York et al. 2010) (Table 1). Inflammatory cell infiltration and demyelination of the CNS in MS/EAE drive clinical disease symptoms and therefore histological assessment of the spinal cord can be crucial in evaluating EAE disease, as well as any past and accurately evaluate MS-like disease within the EAE model has been and will continue to be productive in pushing the MS and CNS inflammatory disease fields forward. 
Key histopathologic findings associated with EAE include inflammatory cell

75 infiltration and axonal loss (Steinbach \& Merkler 2014) which can be identified using

76 routine techniques (Klopfleisch 2013). These include hematoxylin and eosin (HE) and

77 Luxol fast blue (LFB) stains as well as immunohistochemical staining for important

78 inflammatory cell markers such as T cells, B cells and macrophages (Steinbach \&

79 Merkler 2014). This type of histopathologic analysis is commonly performed on EAE-

80 diseased murine spinal cord, which is classically sectioned in a cross-wise (coronal)

81 fashion. While this allows researchers to visualize the entire cord including both white

82 and gray matter, it is at a single level and only approximately $5 \mu \mathrm{m}$ thick. This type of

83 sectioning can be and often is enlightening, however the vast number of spinal cord

84 cross sections makes examination cumbersome. Furthermore, EAE is a multifocal and

85 random disease (Day 2005) and thus it is impossible to predict where lesions will occur

86 throughout the spinal cord, even within areas of expected lesion localization. To

87 histologically evaluate the entire murine spinal cord, we have developed a method to

88 section the cord longitudinally within the decalcified spinal column allowing us to identify

89 and study the multifocal nature of EAE disease.

90

91 MATERIALS AND METHODS

92 Mice

93 C57BL/6 mice (females, 6-8 weeks old) were purchased from The Jackson

94 Laboratory (Bar Harbor, ME). Mice were allowed chow and water ad libitum, maintained

95 on a 12-hour light/dark cycle, and housed in specific pathogen-free barrier facilities at

96 the University of lowa. All animal and tissue work was approved by the University of

97 lowa Institutional Animal Use and Care Committee. 
99

100

101

102

103

104

105

106

107

108

109

110

111

112

113

114

115

116 117 Following euthanasia, the thoracic cavity was immediately opened exposing the heart, 118 the right atrium severed, and a $22 \mathrm{~g}$ needle attached to the gravity flow tubing inserted 119 into the left ventricle. Flow was initiated and the animal perfused with an initial $5 \mathrm{ml}$ of

Immunizations and EAE Evaluation

On day 0 , mice were immunized subcutaneously with $50 \mu \mathrm{g}$ of a myelin oligodendrocyte glycoprotein peptide $\left(\mathrm{MOG}_{35-55}\right)$ emulsified 1:1 volume in CFA supplemented with $4 \mathrm{mg} / \mathrm{mL}$ Mycobacterium tuberculosis (H37Ra; Difco Detroit, MI). Mice were additionally injected intraperitoneally on days 0 and 2 with $250 \mathrm{ng}$ of pertussis toxin (List Biological Laboratories, Campbell, CA). Clinical EAE disease scores were monitored using the grading scale as follows: 1) loss of tail tonicity; 2) mild hind limb weakness; 3) partial hind limb paralysis; 4) complete hind limb paralysis; 5) complete hind limb paralysis with forelimb weakness or moribund/death (Table 1). Specific mice were chosen at various scores for histological evaluation.

\section{Tissue preparation and histology}

Mice were humanely euthanized by carbon dioxide asphyxiation in accordance with NIH \& ACURF guidelines. Rapid fixation was achieved by whole body perfusion with the use of a simple gravity flow device utilizing $10 \%$ neutral buffered formalin (NBF) (Leica Biosystems). A 60cc syringe barrel with attached stopcock \& 3mm diameter tubing was mounted on a ring stand with the syringe $80 \mathrm{~cm}$ above the working surface. The syringe and tubing were flushed with $37^{\circ} \mathrm{C}$ PBS prior to and after each use. 
120 PBS at $37^{\circ} \mathrm{C}$ to clear the vasculature of blood followed by immediate perfusion with 40

$121 \mathrm{ml}$ of $10 \% \mathrm{NBF}$ at $37^{\circ} \mathrm{C}$.

122 Once perfused, the entire spinal column, including the vertebrae and enclosed

123 spinal cord, were removed, epaxial muscles dissected off, and placed in $200 \mathrm{ml}$ of $10 \%$

124 NBF for 3 days at room temperature on an orbital shaker set at 100 RPM for immersion

125 fixation. After 3 days in NBF, the spines were briefly washed with tap water and placed

126 in $200 \mathrm{ml}$ of 14\% EDTA (Sigma ED-EDTA, pH 7.3) for decalcification with continuous

127 shaking. The spines were in 14\% ED-EDTA for 4 days before removal, washed

128 thoroughly with tap water for 3 hours, and sections grossed into cassettes with the use

129 of a microtome blade. The entire spinal column was sectioned in half into longitudinal

130 sections thus exposing the centrally located spinal cord and marking dye was used on

131 the samples to maintain appropriate orientation. Tissues were placed back into $10 \%$

$132 \mathrm{NBF}$, routinely processed, embedded in paraffin, and consecutive sections at $5 \mu \mathrm{m}$

133 thickness were cut for subsequent staining (Figure 1).

134

Routine HE and Luxol fast blue (LFB) staining was performed on all sections.

135 Digital images were collected with a DP73 camera and CellSens software (Olympus).

136 HE-stained, longitudinally sectioned spinal cord sections were evaluated for lesions of

137 EAE, including demyelination and inflammatory cell infiltration. Four separate areas

138 along each spinal cord were identified and in each area the percentage of spinal cord

139 with lesions was estimated visually (using a scale of $0,10,20 \ldots .100 \%$ affected) at $20 x$

140 magnification by a board-certified veterinary pathologist. 


\section{Immunohistochemistry}

144 To validate that immunohistochemistry would be successful using this method of

145 tissue preparation, staining was performed for key inflammatory cells common in EAE

146 (CD3 for $T$ cells, B220 for B cells and F4/80 for macrophages) (Table 2). All

147 immunohistochemical staining was performed manually using peroxidase methods and

148 Dako Envision systems (Glostrup, Denmark).

149

150

\section{RESULTS}

151

HE and LFB staining

152

HE and LFB staining of longitudinally sectioned spinal cord sections (Figure 2)

153

revealed the multifocal to coalescing nature of the lesions associated with EAE. In some

154 areas, the spinal cord can appear relatively normal while in other areas there is

155 significant pathology (Figure 2A, D). When a section of spinal cord from a similarly

156 affected mouse is cut cross-wise, it can be taken from an area with a paucity of lesions

157 (Figure 2B, E), this would lend an investigator to think the cord was relatively

158

unaffected. In contrast, when a cross section is taken within an area of pathology, which

159 includes infiltration of inflammatory cells and demyelination (Figure $2 \mathrm{C}, \mathrm{F}$ ), the severity

160 of disease could be overestimated.

161

Prior to euthanasia, each animal was given a clinical score based on their

162 symptoms of EAE (Table 1). The clinical scores ranged from 2 (mild hind limb

163 weakness) to 4 (complete hind limb paralysis) (Figure 3A). Following necropsy, the

164 percentage of spinal cord affected by EAE lesions was identified at four separate and

165 equidistant areas of the longitudinally sectioned, HE-stained spinal cord sections to

166 determine the variation in lesion severity. Interestingly, the percentage of spinal cord 
167 affected by EAE lesions varied greatly — even within a single sample and that, as

168 expected, the most severe lesions appear to be in more caudal spinal cord (Figure 3B)

169 (Nathoo et al. 2014). This illustrates the utility of longitudinally sectioning EAE spinal

170 cords to increase the odds of identifying lesions.

171

172 Immunohistochemical staining

173 Immunohistochemical staining was performed on these longitudinally sectioned,

174 decalcified specimens (Figure 4). Specific immunomarkers (Table 2) commonly used in

175 EAE research were selected, including CD3 for T cells (Figure 4B), F4/80 for

176 macrophages (Figure 4C) and B220 for B cells (Figure 4D). All successfully stained the

177 decalcified tissues and were able to identify these key immune cells within the EAE 178 spinal cord lesions.

\section{DISCUSSION}

Due to the multifocal nature of EAE within the spinal cord, we developed a novel way of looking at the entire length of the spinal cord in situ, within the vertebrae,

183 allowing us to visualize the multifocal nature of lesions (Figure 1). Using both HE and

184 LFB staining we show that in some areas of the spinal cord there can be significant 185 pathology but in other areas the cord can appear relatively normal (Figure 2). The 186 histopathologic score of the spinal cords somewhat followed the clinical score of these 187 mice antemortem (Figure 3). Interestingly, some animals (mouse 1 and 11) had 188 markedly variable histopathology but had fairly high clinical scores, indicating that 189 clinically there was significant damage to the cord that might be difficult to identify 190 histologically if one did not section the area(s) of cord affected. It is also important to 
191 note that this technique, which uses a decalcification step, also allows for routine

192 immunohistochemical staining for key inflammatory cell infiltrates. Decalcification can

193 affect some immunohistochemical assays but in this case we were able to assess for

194 the key immune cells in EAE; T cells, B cells and macrophages (Figure 4) (Bussolati \& 195 Leonardo 2008).

196 This technique will allow EAE researchers to quickly assess the spinal cord using 197 routine histopathology and without having to make numerous slides of spinal cord cross 198 sections. It is also important to note that the spinal cord itself does not have to be 199 flushed from the spinal column, which not only saves time, but also preserves the 200 integrity of the cord and minimizes handling artifacts within the tissue. Of course, there 201 are limitations. With longitudinal sectioning, one can only visualize a single plane of the 202 cord unlike cross sectioning where all of the white and gray matter can be assessed. If 203 the histotechnologist does not cut deep enough into the tissue, only the white matter will 204 be visible as the deeper gray matter can be missed. It is also difficult to visualize the 205 central canal using longitudinal sectioning. Another challenge is working with the 206 decalcified vertebrae surrounding the softer spinal cord. The spinal column isn't 207 completely flat so the undulating nature of the vertebrae can pose challenges to the 208 histotechnologist during cutting to make sure the spinal cord isn't folded or shredded. 209 In classic EAE the disease presents with hind limb paralysis and the lesions are 210 predominantly seen in the lumbosacral region of the spinal cord, as demonstrated in Fig 211 2. However, it is noteworthy that there are lesions in thoracic and higher segments, 212 which are generally missed in routine evaluation. Moreover, histologic evaluation of 213 EAE using this novel approach will add tremendous value in understanding models of 
214 atypical EAE (such as those in certain knockout mice) or after therapeutic intervention in

215 classic EAE, where certain lesions may resolve and/or re-myelinate, whereas others

216 may not. This is a current gap in the field that could benefit from the methods presented 217 here.

218

219 CONCLUSIONS

220

In summary, longitudinal sectioning of the decalcified spinal column in mice is a

221 useful technique to study the multifocal and oftentimes random nature of spinal cord

222 lesions encountered in EAE. This technique will be useful to the researcher who would

223 like to assess the entire length of the spinal cord without having to remove the spinal

224 cord from the vertebrae and without having to make numerous spinal cord cross

225 sections.

226

227

228

229

230

231

232

233

234

235

236 


\section{REFERENCES}

238

239

240

241

242

243

244

245

246

247

248

249

250

251

252

253

254

255

256

257

258

259

260

261

262

263

264

265

266

267

268

269

270

271

272

273

274

275

276

277
Bussolati G, and Leonardo E. 2008. Technical pitfalls potentially affecting diagnoses in immunohistochemistry. J Clin Pathol 61:1184-1192. 10.1136/jcp.2007.047720

Constantinescu CS, Farooqi N, O'Brien K, and Gran B. 2011. Experimental autoimmune encephalomyelitis (EAE) as a model for multiple sclerosis (MS). Br J Pharmacol 164:1079-1106. 10.1111/j.1476-5381.2011.01302.x

Day MJ. 2005. Histopathology of EAE. In: Ehud Lavi CSC, ed. Experimental Models of Multiple Sclerosis. New York: Springer, 25-43.

Duffy SS, Lees JG, and Moalem-Taylor G. 2014. The contribution of immune and glial cell types in experimental autoimmune encephalomyelitis and multiple sclerosis. Mult Scler Int 2014:285245. 10.1155/2014/285245

Klopfleisch R. 2013. Multiparametric and semiquantitative scoring systems for the evaluation of mouse model histopathology--a systematic review. BMC Vet Res 9:123. 10.1186/1746-6148-9-123

McCarthy DP, Richards MH, and Miller SD. 2012. Mouse models of multiple sclerosis: experimental autoimmune encephalomyelitis and Theiler's virus-induced demyelinating disease. Methods Mol Biol 900:381-401. 10.1007/978-1-60761720-4_19

Nathoo N, Yong VW, and Dunn JF. 2014. Understanding disease processes in multiple sclerosis through magnetic resonance imaging studies in animal models. Neuroimage Clin 4:743-756. 10.1016/j.nicl.2014.04.011

Ortega SB, Kashi VP, Tyler AF, Cunnusamy K, Mendoza JP, and Karandikar NJ. 2013. The disease-ameliorating function of autoregulatory CD8 T cells is mediated by targeting of encephalitogenic CD4 T cells in experimental autoimmune encephalomyelitis. J Immunol 191:117-126. 10.4049/jimmunol.1300452

Sospedra M, and Martin R. 2005. Immunology of multiple sclerosis. Annu Rev Immunol 23:683-747. 10.1146/annurev.immunol.23.021704.115707

Steinbach K, and Merkler D. 2014. Neuropathological Techniques to Investigate CNS Pathology in Experimental Autoimmune Encephalomyelitis (EAE). Methods $\mathrm{Mol}$ Biol. 10.1007/7651_2014_110

Trapp BD, and Nave KA. 2008. Multiple sclerosis: an immune or neurodegenerative disorder? Annu Rev Neurosci 31:247-269. 10.1146/annurev.neuro.30.051606.094313

York NR, Mendoza JP, Ortega SB, Benagh A, Tyler AF, Firan M, and Karandikar NJ. 2010. Immune regulatory CNS-reactive CD8+T cells in experimental autoimmune encephalomyelitis. J Autoimmun 35:33-44. 10.1016/j.jaut.2010.01.003 


\section{TABLES}

279 Table 1. Clinical scoring rubric for EAE.

280

281 Table 2. Primary antibodies and their commercially available sources, catalog 282 numbers, dilutions and specific antigen retrieval conditions utilized in the study.

283

284

285

286

287

288

289

290 


\section{FIGURES}

292 Figure 1. Process of tissue collection and histologic preparation of longitudinally 293 sectioned in situ spinal cord sections.

Figure 2. Photomicrographs of decalcified spinal columns from EAE mice. Longitudinally sectioned spinal cord stained with HE (A) and LFB (D) showing an area which is relatively unaffected (left dotted line) versus one with significant demyelination and inflammatory cell infiltration (right dotted line). The relatively unaffected area corresponds to the HE (B) and Luxol Fast Blue (E) cross sections and the area with lesions corresponds to the HE (C) and Luxol Fast Blue (F) cross sections. Solid arrows indicate areas of inflammation and dotted arrows indicate areas of demyelination. Bars $=200 \mu \mathrm{m}$.

Figure 3. Clinical EAE score and quantification of the percentage of spinal cord affected by EAE using the method of longitudinal sectioning. A. Clinical EAE score (Table 1) of 12 different mice with EAE ranging in age from 7-11 weeks of age. B. Four separate areas (divided equally cranial to caudal) of longitudinally sectioned spinal cord from the same 12 mice, which were evaluated to determine what percentage of the cord at that area was affected by EAE lesions.

Figure 4. Representative images of key immunohistochemical markers run on a longitudinally sectioned, decalcified spinal column from an EAE mouse with a clinical EAE score of 4. A: HE, B: B220 immunohistochemistry for B cells, C: F4/80 immunohistochemistry for macrophages (arrow indicates area highlighted in inset, inset bar $=20 \mu \mathrm{m}$ ), D: CD3 immunohistochemistry for T cells. Bars $=200 \mu \mathrm{m}$. 


\section{Figure 1 (on next page)}

Figure 1.

Process of tissue collection and histologic preparation of longitudinally sectioned in situ spinal cord sections. 

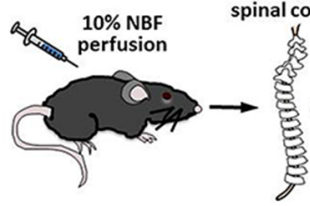

$10 \%$ NBF

3 day soak
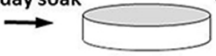

14\% EDTA 4 day decalcification

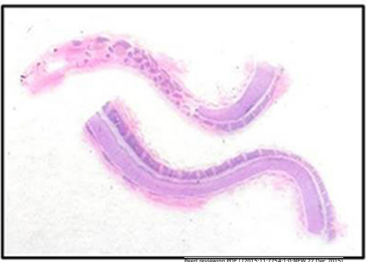

sectioning and staining

longitudinal sectioning of column to expose cord

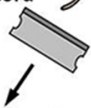

processing and paraffin embedding into cassettes 


\section{Figure 2 (on next page)}

Figure 2.

Photomicrographs of decalcified spinal columns from EAE mice. Longitudinally sectioned spinal cord stained with HE (A) and LFB (D) showing an area which is relatively unaffected (left dotted line) versus one with significant demyelination and inflammatory cell infiltration (right dotted line). The relatively unaffected area corresponds to the HE (B) and Luxol Fast Blue (E) cross sections and the area with lesions corresponds to the HE (C) and Luxol Fast Blue (F) cross sections. Solid arrows indicate areas of inflammation and dotted arrows indicate areas of demyelination. Bars $=200 \mu \mathrm{m}$. 
Figure 3 (on next page)

Figure 3.

Clinical EAE score and quantification of the percentage of spinal cord affected by EAE using the method of longitudinal sectioning. A. Clinical EAE score (Table 1) of 12 different mice with EAE ranging in age from 7-11 weeks of age. B. Four separate areas (divided equally cranial to caudal) of longitudinally sectioned spinal cord from the same 12 mice, which were evaluated to determine what percentage of the cord at that area was affected by EAE lesions. 


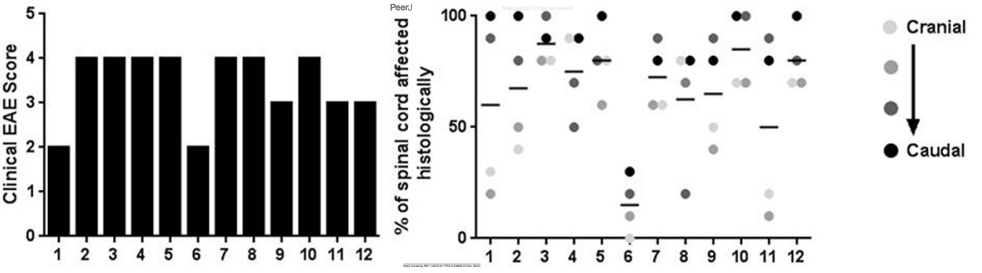




\section{Figure 4 (on next page)}

Figure 4.

Representative images of key immunohistochemical markers run on a longitudinally sectioned, decalcified spinal column from an EAE mouse with a clinical EAE score of 4. A : HE, B : B220 immunohistochemistry for $B$ cells, $C$ : F4/80 immunohistochemistry for macrophages (arrow indicates area highlighted in inset, inset bar $=20 \mu \mathrm{m}), \mathrm{D}:$ CD3 immunohistochemistry for T cells. Bars $=200 \mu \mathrm{m}$. 

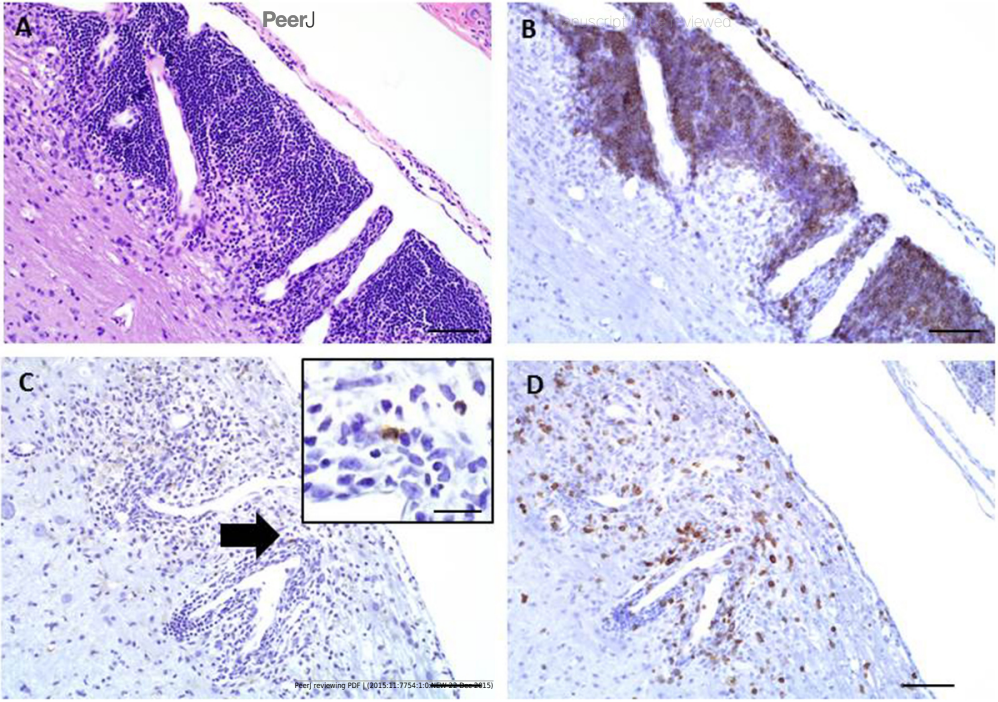


\section{Table 1 (on next page)}

Table 1 


\begin{tabular}{cl}
\hline Score & \multicolumn{1}{c}{ Clinical findings } \\
\hline $\mathbf{1}$ & loss of tail tonicity \\
$\mathbf{2}$ & mild hind limb weakness \\
$\mathbf{3}$ & partial hind limb paralysis \\
$\mathbf{4}$ & complete hind limb paralysis \\
$\mathbf{5}$ & complete hind limb paralysis with forelimb weakness or moribund \\
\hline
\end{tabular}




\section{Table 2 (on next page)}

Table 2 


\begin{tabular}{ccccc}
\hline Marker & Antibody & Dilution & Source & Conditions \\
\hline CD3 & Cat\# RM-9107-5 & $1: 200$ & Neomarkers & HIER, citrate buffer $(\mathrm{pH} 6.0)$ \\
B220 & Cat\# MCA1258G & $1: 6000$ & Serotec & HIER, citrate buffer $(\mathrm{pH} \mathrm{6.0)}$ \\
F4/80 & Cat\# MCAP497 & $1: 6400$ & Serotec & HIER, citrate buffer (pH 6.0) \\
\hline
\end{tabular}

